\title{
Efecto de la lluvia ácida en el material constituyente de los monumentos mayas mexicanos
}

\author{
H. Bravo-Álvarez, R. Soto-Ayala, R. Sosa-Echeverría y P. Sánchez-Álvarez \\ Sección de Contaminación Ambiental, Centro de Ciencias de la Atmósfera, UNAM \\ División de Ciencias Básicas, Facultad de Ingeniería, UNAM \\ E-mails:hbravo@servidor.unam.mxyrsoto54@hotmail.com
}

(recibido: marzo de 2003; aceptado: julio de 2003)

\section{Resumen}

Actualmente la lluvia ácida se ha convertido en un problema potencial que afecta en mayor o menor grado a materiales y a los ecosistemas. El efecto que causa la lluvia ácida en monumentos construidos fundamentalmente de roca caliza, ha sido objeto de muchas investigaciones realizadas, prioritariamente, por países que poseen estructuras, cuyo material de construcción es caliza. En este trabajo de investigación se estudió una muestra de roca caliza proveniente de los monumentos mayas de Tulum, Quintana Roo. Se determinaron los parámetros de densidad, porosidad y porcentaje de adsorción de agua. Asimismo, se efectuó la irrigación de la muestra con lluvia ácida ( $\mathrm{pH}$ aprox.4.6), preparada en el laboratorio con base en la composición química que se determinó en 56 muestras de agua de lluvia provenientes de Puerto Morelos, punto cercano a los monumentos de Tulum, Quintana Roo, durante los años 1994 y 1995.

Los resultados permiten concluir que la lluvia ácida sufre un proceso de neutralización con el carbonato de calcio de la roca caliza. Las altas concentraciones de calcio en el efluente, mostraron que la roca caliza sufrió una disolución con la lluvia ácida. La recesión superficial promedio que sufrió la roca, de acuerdo con las condiciones experimentales utilizadas en este trabajo, fue $4.4 \mu \mathrm{m} / \mathrm{año}$.

Descriptores: lluvia ácida, efecto en materiales, deterioro, mayas, recesión superficial.

\begin{abstract}
Actually, acid rain is considered as a potential problem that affects materials and ecosystems. The effect on monuments, made mainly from limestone, has been studied for long time. In this work, a sample of limestone from Tulum, Quintana Roo, was studied. The following parameters were measured: density, porosity and percentage of water adsorption. Also, the sample was irrigated with acid rain prepared in the laboratory ( $p H=4.6)$, based in the chemical composition of 56 rain samples from Puerto Morelos, Quintana Roo, in 1994 and 1995.

The results obtained show that acid rain is neutralized by calcium carbonate from the limestone. The high calcium concentrations in the effluent, show that limestone is dissolved by acid rain. Superficial recession was $4.4 \mu \mathrm{m} /$ year under experimental conditions used.
\end{abstract}

Keywords: acid rain, effects on materials, deterioration, mayas, superficial recession. 


\section{Introducción}

El proceso mediante el cual algunos compuestos se depositan por medio de la lluvia o la nieve sobre las superficies de los materiales, se llama deposición húmeda. Sin embargo, también ocurre otro proceso atmosférico conocido como deposición seca. En la deposición seca, partículas tales como cenizas, o gases, como el dióxido de azufre $\left(\mathrm{SO}_{2}\right)$ o el óxido nítrico (NO), se depositan o se adsorben sobre las superficies de los materiales. Los mecanismos precisos por los cuales la deposición seca se lleva a cabo y sus efectos sobre los suelos, bosques, cosechas y edificios, aún no se entienden totalmente.

En cuanto a la deposición húmeda, esta se presenta cuando algunos compuestos, sobre todo los contaminantes sulfurados y nitrogenados al entrar en contacto con el agua, dan lugar a los ácidos sulfúrico y nítrico, respectivamente. Estos ácidos fuertes disminuyen el $\mathrm{pH}$ de la lluvia a un valor inferior de 5.6 (valor del pH de la lluvia en equilibrio con el dióxido de carbono atmosférico). A la lluvia con estas características se le aplica el nombre genérico de lluvia ácida.

Todos los materiales expuestos a" cielo abierto" son susceptibles a deteriorarse por efecto de la lluvia ácida; sin embargo, uno de los aspectos a nivel mundial al que se le ha prestado más importancia, es al efecto que causa la lluvia ácida sobre materiales que forman parte del patrimonio cultural de una nación, como son en México los monumentos mayas.

En las sociedades industriales contemporáneas los procesos de intemperismo natural se han agudizado por las concentraciones elevadas de los contaminantes, tanto en el aire como en la lluvia, particularmente los ácidos, que están identificados entre los problemas más serios que afectan a las rocas calizas, componentes fundamentales de los monumentos mayas.

En general, los ácidos presentes en las deposiciones secas y húmedas, son agentes importantes que causan deterioro en la roca caliza y en el mármol. El daño que se genera puede ser el resultado de la reacción ácida directa con la superficie de la roca, o se puede deber a la formación de fases minerales secundarias, tales como el yeso $\left(\mathrm{CaSO}_{4} 2 \mathrm{H}_{2} \mathrm{O}\right)$, que es un producto de la acción del ácido sulfúrico sobre la roca. Así también, el transporte capilar del sulfato de calcio a través de los poros de la roca puede causar un incremento en el volumen de ésta y en consecuencia acelerar su deterioro.

La temperatura, la humedad y el dióxido de carbono en la solución que está en contacto con el material, son otros de los factores que influyen en su deterioro.

Por lo tanto, conocer los mecanismos detallados del daño que sufre la roca caliza en un ambiente contaminado es fundamental para proteger y conservar las estructuras del efecto de la lluvia ácida y de la contaminación del aire.

En México existen relativamente pocos estudios concernientes a evaluar el efecto que causan los contaminantes atmosféricos sobre los monumentos mayas. No se han reportado investigaciones que hagan uso de cámaras de intemperismo acelerado donde se simule el efecto de degradación que causa la lluvia ácida sobre estos materiales.

La presente investigación tiene como propósito cuantificar los efectos que causa la lluvia ácida en el material con el que están construidos los monumentos mayas, 
legado invaluable de la cultura de México y de América Central; esto se logró mediante la utilización de una cámara de intemperismo acelerado (CIA). Asimismo, se pudieron establecer los mecanismos de disolución que intervienen en el proceso de deterioro de tales monumentos, mediante la caracterización precisa del material utilizado en su construcción.

\section{Antecedentes}

Se han realizado algunos estudios referentes al impacto que causa la lluvia ácida sobre algunos materiales. Cheng y Castillo (1984) llevaron a cabo un estudio sobre el deterioro del mármol del City Hall, en Schenectady, New York. Ellos encontraron que la conversión de mármol en cristales de yeso se debe a la existencia de Fe (III) que actúa como catalizador. El espesor de mármol que sufre esta conversión química es de 2 a $5 \mathrm{~mm}$, aproximadamente, y la estructura del mármol se debilita debido a este proceso. Asimismo, se determinó que otro de los factores que influye en el deterioro del mármol es la presencia de partículas carbonáceas con alto contenido de azufre.

Por otra parte, Baer et al. (1986), en una conferencia internacional que se llevó a cabo en Amsterdam, puntualizaron que los óxidos de azufre $\left(\mathrm{SO}_{\mathrm{x}}\right)$ y otros gases ácidos son los responsables de la erosión superficial del material de construcción; sin embargo, manifiestan que el proceso de degradación atmosférica es un fenómeno complejo, gobernado por muchos factores relacionados entre sí, y de los cuales, la contaminación del aire es sólo uno de ellos. Asimismo, indican que el análisis de los procesos de deterioro, inducido por factores meteorológicos, se debe enfocar desde el punto de vista de la termodinámica y de la transferencia de calor. También aseguran que la humedad de la atmósfera es un factor muy importante que se debe tomar en consideración.

Se ha comentado (Lipfert, 1987) el uso de cámaras de intemperismo acelerado para estudiar los efectos que causan algunas mezclas de aire contaminado sobre materiales, tales como metales y material calcáreo. Se asegura también que esta técnica, efectivamente, permite la determinación de cada contaminante por separado; no obstante, menciona que es difícil simular los niveles de turbulencia de la atmósfera, así como los efectos de la precipitación natural, por lo que se debe tener cuidado en extender los resultados cuantitativos obtenidos en las cámaras a la atmósfera real.

Reddy y Youngdahl (1987) expusieron algunas muestras de roca a las condiciones atmosféricas en cuatro sitios al este de los Estados Unidos. La exposición duró dos años y al final se encontró que la recesión superficial, definida como el espesor de la roca que sufrió daño, para muestras de mármol en Carolina del Norte, fue de 15 $\mu \mathrm{m} / a$ ño. La recesión superficial se incrementó con la cantidad de lluvia incidente, así como con el aumento de la acidez de la lluvia, aunque este último efecto fue menos importante que el primero.

Baedecker et al. (1992), realizaron pruebas de deposición ácida en cinco sitios de los Estados Unidos, autorizados por el programa NAPAP en 1984, para llevar a cabo investigaciones sobre efectos en metales, pinturas y material calizo. 
Para estimar la recesión superficial de la roca caliza, los parámetros que se consideraron fueron: la cantidad de lluvia incidente, la concentración total de calcio en el efluente, el área de la muestra sobre la que incidió la lluvia, la densidad de la roca y la pérdida de agua por evaporación que sufrió la muestra.

Los resultados indicaron una recesión superficial de 6 a $10 \mu \mathrm{m} / \mathrm{año}$ y concluyen, que alrededor del $70 \%$ de la disolución de la roca se debe a la solubilidad de la calcita en agua, en equilibrio con el dióxido de carbono atmosférico.

Reddy (1988) puntualiza en otra investigación que se llevó a cabo en el noreste de los Estados Unidos a finales de 1984, que la disolución de la superficie de la roca aumenta la concentración de calcio en el efluente. Este incremento en la concentración de calcio se puede traducir en una pérdida superficial de carbonato de calcio. No se encontraron diferencias notables entre las recesiones superficiales exhibidas entre la roca caliza y el mármol. Los resultados presentados en dicho trabajo muestran que la recesión superficial es directamente proporcional a la concentración de ion hidrógeno que incide sobre la superficie de la roca, así como a la cantidad de lluvia incidente.

Peleg et al. (1989), elaboraron un estudio sobre el deterioro que causan los contaminantes del aire sobre roca caliza de Jerusalem. Para ello, dichas muestras se expusieron a altos niveles de contaminantes $\left(\mathrm{SO}_{2}, \mathrm{NO}\right)$ en la presencia y en la ausencia de hidrocarburos, vapor de agua y luz ultravioleta. Después de la exposición, las capas externas de las muestras se rasparon y se analizaron para determinar sulfatos y nitratos. Posteriormente, los resultados revelaron que a pesar de un solo día de exposición se encontraron concentraciones significativas de $\mathrm{CaSO}_{4}$ y $\mathrm{Ca}$ $\left(\mathrm{NO}_{3}\right)_{2}$ hasta una profundidad de $40 \mu \mathrm{m}$.

En México, donde existe un número apreciable de estructuras que están sujetas a daños irreversibles, es de vital importancia cuantificar el daño a los materiales, particularmente en aquellas estructuras de interés histórico o cultural, que ya presentan un deterioro apreciable debido a los niveles de contaminación del aire en el pasado.

\section{Metodología}

Las muestras de roca caliza utilizadas en el experimento se obtuvieron cerca de Tulum $\left(20^{\circ} 12^{\prime} \mathrm{N} ; 87^{\circ} 27^{\prime} \mathrm{W}\right)$, un sitio arqueológico maya localizado al noroeste de Quintana Roo, México. La figura 1 indica la ubicación del sitio de muestreo.

La temperatura y la humedad relativa del lugar varían de una época del año a otra; sin embargo, para esta investigación se consideraron los valores de $26^{\circ} \mathrm{C}$ y $80 \%$ respectivamente, los cuales representaron aproximadamente los valores promedios de dichas variables en el lugar.

Asimismo, se determinaron otras características físicas de la roca, como la densidad y la porosidad (de acuerdo con los procedimientos indicados en ISRM (1979) y RILEM (1980). El porcentaje de ad sorción de agua se determinó de acuerdo con el estándar de la ASTM (1985), C97-47.

Antes de su irrigación con lluvia ácida, las muestras de roca caliza se cortaron, pulieron y limpiaron para eliminar todo material extraño que pudiera provocar la contaminación del agua que incidiría sobre ellas. Para conocer la composición de la roca 
caliza, a una porción del material se le realizó el análisis de difracción por rayos -x.

El sitio de muestreo del agua de lluvia está localizado en la Estación de Biología Marina de Puerto Morelos ( $20^{\circ} 5^{\prime} \mathrm{N}$; $86^{\circ} 54^{\prime}$ W) , perteneciente al Instituto de Ciencias del Mar y Limnología de la Universidad Nacional Autónoma de México (Figura 1). El sitio cumple con los criterios de selección establecidos por la EPA, en cuanto a satisfacer las normas para ser considerado como un sitio de muestreo de precipitación tipo regional.
Es pertinente comentar que Puerto Morelos es un pueblo pequeño localizado aproximadamente a $95 \mathrm{~km}$ al noreste de Tulum, y a $50 \mathrm{~km}$ al norte de la isla de Cozumel, donde se localiza una planta termoeléctrica cuyo combustible es combustóleo con alto contenido de azufre.

El colector de precipitación húmeda (marca Aeruchem Metrics Collector) está ubicado en una zona campestre para evitar la contaminación de origen local (Figura 2). Este sitio está localizado a más de $50 \mathrm{~m}$ de la playa.

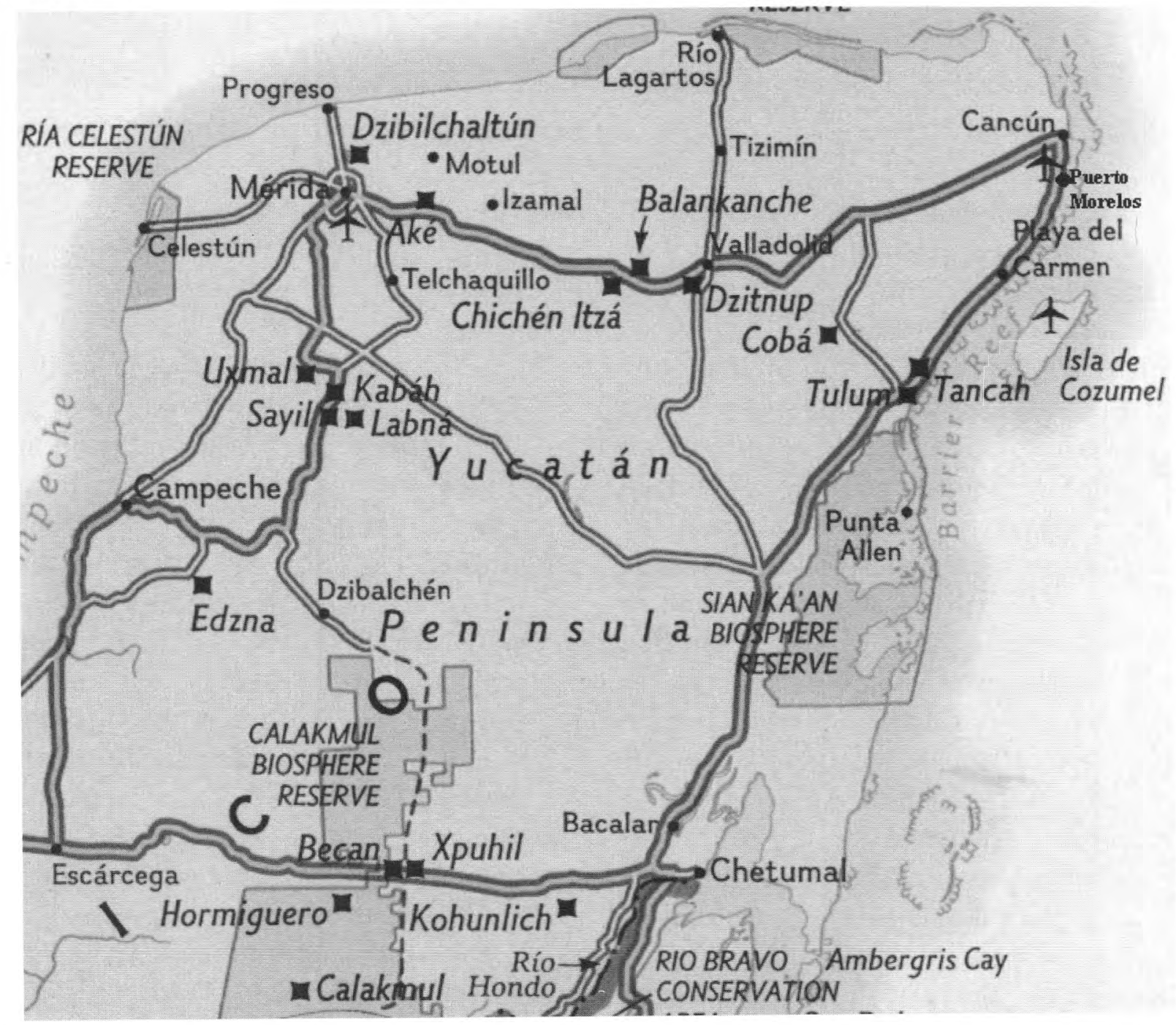

Figura 1. Ubicación del sitio de muestreo de la roca caliza (Tulúm) y de las muestras de agua de lluvia (Puerto Morelos) 


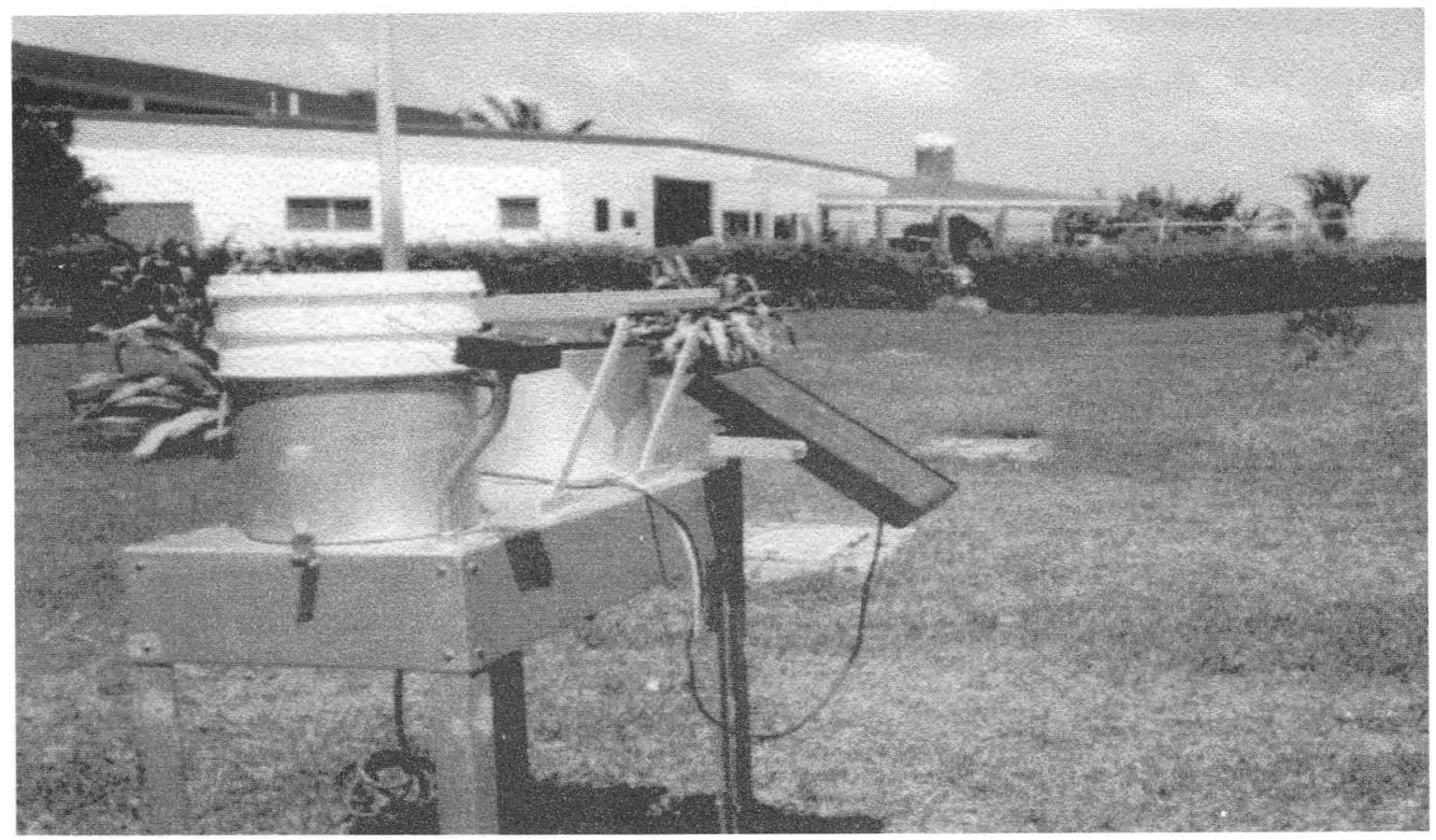

Figura 2. Colector automático de precipitación húmeda y seca en Puerto Morelos, Quintana Roo

Para la recolección de las muestras se contó con el apoyo del personal del Instituto de Ciencias del Mar y Limnología.

Durante un período de dos años (1994-1995) se recolectaron muestras de agua de lluvia en Puerto Morelos, para ello se utilizaron recipientes de polipropileno (Nalgene). Inmediatamente después de la recolección se midió el volumen de precipitación, así como el pH de cada una de las muestras (utilizando un pHmetro Corning, modelo 3D), después se taparon los recipientes perfectamente y las muestras se colocaron en refrigeración a $4^{\circ} \mathrm{C}$, de acuerdo con las recomendaciones de la EPA (1994), en donde se especifica la recolección y manejo de las muestras. Finalmente, las muestras de agua se enviaron periódicamente a la Ciudad de México para su análisis químico en el laboratorio de Ciencias de la Atmósfera.

En el laboratorio se les determinó nuevamente el pH, así como la conductividad. Las concentraciones iónicas de
$\mathrm{Na}^{+}, \mathrm{NH}_{4}^{+}, \mathrm{K}^{+}, \mathrm{Mg}^{2+}, \mathrm{Ca}^{2+}, \mathrm{Cl}^{-}, \mathrm{NO}_{3}{ }^{-} \mathrm{y}$ $\mathrm{SO}_{4}{ }^{2-}$, se cuantificaron mediante un cromatógrafo de líquidos de alta resolución.

En cuanto a la cámara de intemperismo acelerado, ésta se construyó de acrílico y se diseñó tomando en consideración que las condiciones de temperatura y humedad deberían ser prácticamente constantes durante el experimento. Para mantener la temperatura constante, se utilizó una lámpara de luz infraroja, cuya intensidad se graduaba con un dimmer. Así también, en cuanto a la alta humedad relativa que se requería, ésta se alcanzaba al controlar la rapidez de evaporación de cierta cantidad de agua dentro de la cámara. En la figura 3 se muestra la cámara de intemperismo acelerado utilizada para esta investigación.

La composición química del agua de lluvia proveniente de Puerto Morelos sirvió como base para la preparación en el laboratorio del agua de lluvia artificial, la cual se hizo incidir sobre las muestras de roca caliza. 


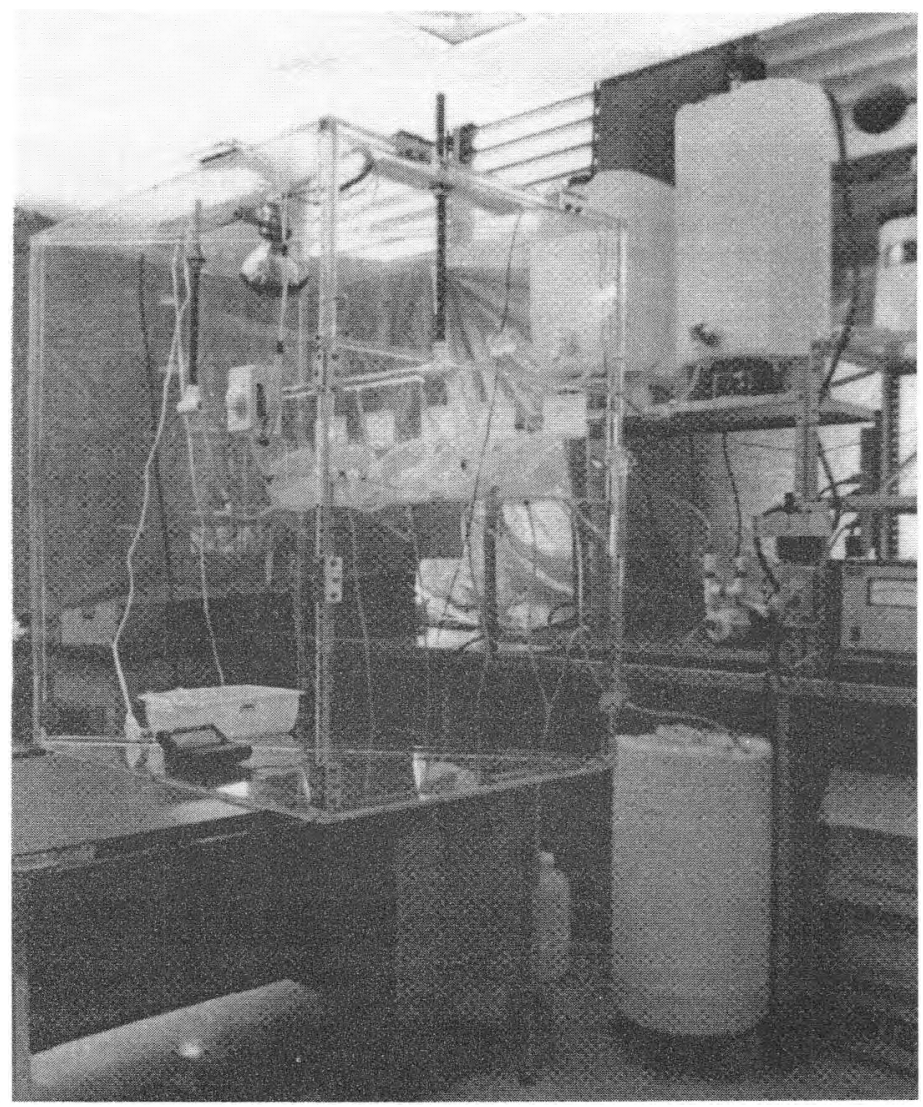

Figura 3. Cámara de intemperismo acelerado para estudios de deposición ácida

Se eligió preparar el agua al pH más ácido que se determinó en las muestras de agua de lluvia durante los dos años de muestreo, esto con el objeto de acelerar el deterioro de la roca caliza (intemperismo acelerado).

La composición iónica, tanto del agua que incidió sobre las muestras de roca caliza, como del efluente, se determinó mediante la cromatografía de líquidos de alta resolución.

Finalmente, se determinó la recesión superficial de la roca caliza, esto es, el deterioro superficial que sufrió el material como producto de la disolución por la lluvia ácida.

\section{Resultados obtenidos}

En la figura 4 se indican los valores de los pH's de las muestras de agua de lluvia colectadas en Puerto Morelos durante los años 1994 y 1995. Como se observa en la figura, el menor valor de $\mathrm{pH}$ para ambos años fue de 4.6. La lluvia ácida que se preparó en el laboratorio para la irrigación de las muestras, se ajustó a este valor con el objeto de favorecer aún más la disolución del material.

Por otra parte, la tabla 1 muestra los valores de densidad, porosidad y adsorción de agua de las muestras de roca caliza.

La recesión superficial de cada una de las muestras de roca caliza se determinó tomando en consideración los parámetros siguientes: la concentración total neta de calcio en el efluente, el volumen total de agua ácida incidente, el área de la roca, su densidad y su porosidad.

Los resultados obtenidos se muestran en la tabla 2. 


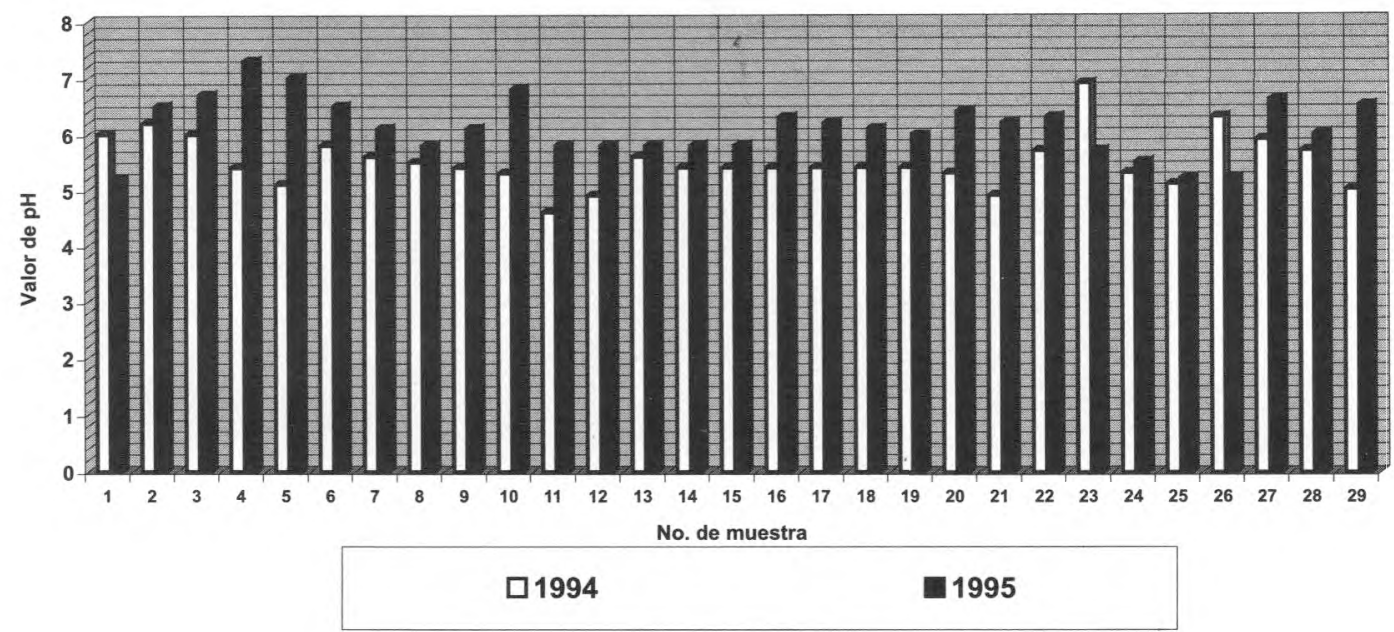

Figura 4. Valores de $\mathrm{pH}$ de las muestras de agua de lluvia colectadas en Puerto Morelos, Quintana Roo, durante los años de 1994 y 1995

Tabla 1. Valores de densidad y adsorción de agua de la roca caliza

\begin{tabular}{ccc}
\hline Densidad $\left(\mathrm{g} / \mathrm{cm}^{3}\right)$ & Porosidad $(\%)$ & Adsorción de agua $(\%)$ \\
\hline $2.3 \pm 0.05$ & $10.7 \pm 0.2$ & $9.2 \pm 0.2$ \\
\hline
\end{tabular}

Tabla 2. Valores de la recesión superficial de la roca caliza

\begin{tabular}{ccc}
\hline Muestra No. & Recesión Superficial, $\mu \mathrm{m} /$ año \\
\hline 1 & $4.71 \pm 0.19$ \\
2 & $4.21 \pm 0.29$ \\
3 & $4.30 \pm 0.16$ \\
\hline
\end{tabular}

\section{Conclusiones}

1. - La cromatografía de líquidos de alta resolución, mostró ser una técnica analítica muy útil y confiable en la cuantificación de los aniones y de los cationes en las muestras de agua de lluvia.

La determinación conjunta en una sola corrida, tanto de aniones como de cationes, permite un ahorro considerable de tiempo, aunado a la facilidad de preparación de las muestras previas a su determinación.
2.- El agua delluvia procedente de Puerto Morelos, Quintana Roo en el año de 1994, presentó características más ácidas que en el año de 1995. Se observó que de los 28 datos presentes en el año de 1994, 20 de ellos poseen un valor inferior a 5.6; mientras que sólo 4 de los 28 datos correspondientes a 1995, cumplen con esta característica.

3. - Con base en el análisis de difracción por rayos-x, la muestra de roca caliza 
utilizada en la construcción de los monumentos mayas contiene, efectivamente, como compuesto mayoritario carbonato de calcio en forma de calcita y de aragonita. Asimismo, contiene un material arcilloso: montmorillonita.

4.- La roca caliza que se utilizó en esta investigación tuvo un contenido de carbonato de calcio de $(87 \pm 0.5)^{\circ} \%$.

5.- El catión cuya concentración es mayoritaria en el efluente es el calcio. Esto es evidente, ya que el carbonato de calcio contiene el $40 \%$ en masa de este elemento, el cual es disuelto y eluido por la lluvia ácida al incidir sobre el material.

6.- Debido a que en el efluente, se encuentran altas concentraciones de iones bicarbonato $\left(\mathrm{HCO}_{3}{ }^{-}\right)$, al llevarse a cabo la neutralización del agua ácida por el carbonato de calcio, se puede sugerir que el mecanismo de disolución químico de la roca es el siguiente:

$$
\mathrm{CaCO}_{3}+\mathrm{H}^{+} \rightarrow \mathrm{HCO}_{3}+\mathrm{Ca}^{2+}
$$

Dicho mecanismo se ve favorecido aún más por los resultados experimentalmente encontrados, ya que son congruentes con la estequiometría de tal ecuación química.

7.- La recesión superficial promedio de la roca caliza utilizada en esta investigación tuvo un valor de $4.4 \mu \mathrm{m} / \mathrm{año}$.

\section{Evaluación de resultados}

1.- Baedecker et al. (1992), reportan valores de recesión superficial para roca caliza entre 6.7 y $9.0 \mu \mathrm{m} / \mathrm{año}$. En este trabajo, el intervalo de valores se encuentra entre 4.0 y $5.0 \mu \mathrm{m} / \mathrm{año}$, aproximadamente. Sin embargo, es importante mencionar que ambos experimentos discrepan en lo siguiente: dichos investigadores exponen las muestras de roca caliza a la lluvia natural más que a la lluvia preparada artificialmente en el laboratorio, utilizan factores empíricos para relacionar la cantidad de agua que incide sobre las muestras y del efluente que se obtiene, y asignan un valor teórico de porosidad a la roca que difiere sustancialmente del utilizado en esta investigación (0.36 vs 0.11 ).

2.- Es importante mencionar que la degradación de la roca caliza no proviene exclusivamente de factores químicos, sino también de aquellos de origen físico, bacteriológico, etc., siendo algunos de ellos más importantes que otros. Esta observación permite asegurar que la recesión superficial real de la roca es mayor que la calculada.

La investigación realizada en este trabajo es sólo la parte de un proyecto más global, el cual abarcaría como segunda etapa el estudio de materiales consolidantes que se pudieran aplicar a la roca para minimizar su deterioro, al incidir la lluvia ácida sobre ella.

La utilización de materiales poliméricos es una opción que resulta muy prometedora, ya que algunos investigadores en esta área la han explotado y continúan haciéndolo.

\section{Agradecimientos}

Los autores agradecen el apoyo para la realización de los análisis químicos de las muestras de agua a las Químicas Ma. Isabel 
Saavedra R. y Ana Luisa Alarcón J., así como a Jaime Ruiz Barrios, pasante de la carrera de ingeniero civil, por su apoyo en la realización de este trabajo.

\section{Referencias}

ASTM (1985). Standard Methods of Test for Absorption and Bulk Specific Gravity of Natural Building Stone. No. C97-47. American Society for Testing Materials. Part XVI, 1-3.

Baedecker P.A. et al. (1992). Effects of Acidic Deposition on the Erosion of Carbonate Stone Experimental Results from the U.S. National Acid Precipitation Assessment Program (NAPAP). Atmospheric Environment, 26B, No. 2, 147-158.

Baer S.N. et al. (1986). Effects of Acidification of Materials and Cultural Property. Studies in Environmental Science 30. Acidification and its Policy Implications. Proceedings of an International Conference Held in Amsterdam, may 5-9, 189-213.
Cheng J.R. y Castillo R. (1984). A Study of Marble Deterioration at City Hall. Schenectady, New York.

ISRM (1979). Commision on Standarization of Laboratory and Field Test. Int. J. Rock Mech. Min. Sci. $\theta$ Geomech. Abstr. 16, 2, 143-156.

Lipfert W.F. (July 1987). Effects of Acidic Deposition on the Atmospheric Deterioration of Materials. Materials Performance. Peleg M. et al. (1989). Deterioration of Jerusalem Limestone from Air Pollutants; Field Observations and Laboratory Simulation. Environmental Monitoring and Assessment.

Reddy M.M. (1988). Acid Rain Damage to Carbonate Stone: A Quantitative Assessment Based on the Aqueous Geochemistry of Rainfall Runoff from Stone. Earth Surface Processes and Landforms.

Reddy M.M. y Youngdahl A.C. (July 1987) Acid Rain and Weathering Damage to Carbonate Building Stone. Materials Performance.

RILEM (1980). Essais Recommande's pour Mesurer I' Alteration des Pierres et Evaluer I 'Efficacité des Métodes de Traitement. Materiaux et Constructions. 


\section{Semblanza de los autores}

Dr. Humberto Bravo-Álvarez. Realizó su licenciatura en química en la UNAM, así como la maestría en química nuclear. Posteriormente, obtuvo el grado de doctor en ingeniería en la Universidad de West Virginia. Es investigador en el Centro de Ciencias de la Atmósfera (CCA) de la UNAM, donde se desempeña como jefe de la Sección de Contaminación Ambiental desde 1977. Su área de interés por más de 30 años ha sido la contaminación y la ingeniería ambiental. Ha sido profesor de asignatura en la maestría en ingeniería ambiental en la División de Estudios de Posgrado de la Facultad de Ingeniería (DEPFI) de la UNAM desde 1988. Es perito colegiado del Colegio Nacional de Ingenieros Químicos y de Químicos (CONIQQ) en protección ambiental, así como Qualified Environmental Professional (QEP) del Institute of Professional Environmental Practice.

Dr. Rogelio Soto-Ayala. Realizó su licenciatura en química y su maestría en química analítica en la Facultad de Química de la UNAM. Obtuvo el doctorado de ingeniería ambiental en la División de Estudios de Posgrado de la Facultad de Ingeniería de la UNAM (DEPFI). Tiene 22 años de experiencia docente en la Facultad de Ingeniería y actualmente participa en actividades de investigación en el CCA.

Dr. Rodolfo Sosa-Echeverría. Realizó su licenciatura en ingeniería química, la maestría en ingeniería ambiental y el doctorado en ingeniería en la UNAM. Labora en la Sección de Contaminación Ambiental del CCA desde 1986 y ha impartido cátedra en la DEPFI desde 1988. Es perito colegiado del Colegio Nacional de Ingenieros Químicos y de Químicos (CONIQQ) en protección ambiental y fue presidente del Capítulo México de la Air Waste Management Association, de 1991 a 1999.

Pablo Sánchez-Álvarez. Realizó la licenciatura en biología en la Facultad de Ciencias de la UNAM. Actualmente labora en la Sección de Contaminación Ambiental del CCA. Es responsable de la operación y mantenimiento de la estación de monitoreo automático de contaminantes criterio y deposición ácida. Es instructor certificado en la línea de investigación de la atmósfera y el clima del Programa Internacional de Aprendizajes y Observaciones Globales en Beneficio del Medio Ambiente (The GLOBE Program México). 